\title{
Review of: "Neurocognitive outcomes following fetal exposure to chemotherapy for gestational breast cancer: A Canadian multi-center cohort study"
}

\author{
Evangeline Huis in 't Veld ${ }^{1}$, Mathilde van Gerwen', Frédéric Amant ${ }^{2}$ \\ 1 Princess Máxima Center for pediatric oncology \\ 2 Netherlands Cancer Institute
}

Potential competing interests: The author(s) declared that no potential competing interests exist.

\begin{abstract}
Evangeline Huis in 't Veld ${ }^{1,2}$, Mathilde van Gerwen ${ }^{1,2}$, Frédéric Amant ${ }^{1,3}$ on behalf of the International Network on Cancer, Infertility and Pregnancy (INCIP)
\end{abstract}

1 Center for Gynecologic Oncology, The Netherlands Cancer Institute and Amsterdam University Medical Centers, Amsterdam, the Netherlands

2 Princess Máxima Center for pediatric oncology, Utrecht, the Netherlands

3 Department of Oncology, KU Leuven, Leuven, Belgium

This article represents a well-performed study in multiple Canadian centers and describes extensively the neurocognitive outcomes in 17 children of Breast Cancer (BC) patients who were treated during pregnancy and their matched controls. The principal finding was that fetal exposure to chemotherapy for BC was not neurotoxic for the offspring. The authors have adjusted for several possible confounders. In the current review we would like to discuss some of our considerations in order to put the results of this study in its perspective.

Firstly, the authors mention the use of two age-appropriate intelligence tests: the WPPSI-III and the WISCIV. These tests are both standardized indeed, but the 2-7-yr age-range of the participants in this study is wide. An age-appropriate standardized intelligence test (WPPSI-III) in 2-year olds will (clinically) assess other outcomes than an age-appropriate standardized intelligence test in 7-year olds (WISC-IV). Besides, 7-year olds are used to an school setting and therefore more used to formal assessment procedures while 2-year olds are mostly not able yet to sit quiet and perform structured tasks of an intelligence test. This can cause bias in the intelligence outcomes, also a longitudinal aspect was not measured.

Moreover, greater nuance is required to the conclusion of the absence of neurotoxicity in the offspring, because of the 'growing into deficit hypothesis'. This hypothesis implies that the extent and range of one's 
'impairments' may not be identified initially, but are exposed as the demands of the environment (such as school subjects, capacities of planning and organization) increase. This explains the possibility of neurotoxicity in later ages which cannot be ruled out based on this study and the short follow-up period.

Furthermore, the study is performed over a seven-year period, a period of several societal changes. Additionally, as well the WPPSI as the WISC intelligence tests were updated in this period, because of contemporary structural intelligence theories. Therefore, we suggest some extra information about the years in which the tests were administered. Besides, the authors should argue why they did not opt for the updated intelligence tests, such as the WISC-V.

Another point of consideration is the influence of the stressful pregnancy on the neurocognition of the offspring. Two life-events at the same time, like a pregnancy and a cancer diagnosis can cause a burden of stress in the parents. Maternal conditions, such as stress, during the first 1000 days can develop neuropsychological disorders later in life. We suggest this can be an interesting consideration for the discussion.

In conclusion, the paper describes a unique series with an important finding: absence of neurotoxicity in the offspring of BC patients. We think that a nuance is required. Indeed, the results of the study are indicative for the absence of severe neurotoxic problems in the offspring, but those results should be interpreted carefully and further research is absolutely needed to support these findings. Since 2005, a similar project has been ongoing by the International Network on Cancer, Infertility and Pregnancy (INCIP, www.cancerinpregnancy.org). They study the effects of various cancer therapies on the health of both mother and child in an innovative research project. The authors could consider collaboration with INCIP allowing them to help building on a large dataset. Only such comprehensive sets of data are able to deliver clinically sound results that will assist patients and physicians during the decision making process. 